\title{
Funciones generadoras en Geometría Simpléctica
}

\section{Josué Alonso Aguirre Enciso ${ }^{1}$ y Rodolfo José Gálvez Pérez ${ }^{2}$}

Resumen: En este trabajo presentamos una breve introducción a la Geometría Simpléctica mostrando su origen relacionado con la Física. Luego presentamos la definición formal de variedades simplécticas y algunos resultados importantes, para ello consideramos una función $\mathbb{A}_{H, N}$ definida en el producto cartesiano de la variedad simpléctica $\left(\mathbb{R}^{2 n}, \omega_{0}\right)$ y hacemos un análisis con el comportamiento de esta función de donde concluimos que los puntos críticos de esta función se relacionan de manera biunívoca con los puntos fijos del flujo $\phi^{t}$ de la variedad Simpléctica $\left(\mathbb{R}^{2 n}, \omega_{0}\right)$ en el tiempo $t=1$ esto debido a las ecuaciones diferenciales Hamiltonianas via las funciones generadoras.

Palabras clave: Variedad Simpléctica, Funciones Generadoras, Campos vectoriales, Simpléctomorfismo.

\section{Generating functions in Symplectic Geometry}

\begin{abstract}
In this work, we present a brief introduction to Symplectic Geometry relating its origin with the Physics. Then we present the formal definition of symplectic manifold and some important results, with this we consider a function $\mathbb{A}_{H, N}$ defined in the Cartesian product of the symplectic manifold $\left(\mathbb{R}^{2 n}, \omega_{0}\right)$. Here we make an analysis with the fact that the critical points of this function are related in a biunivocal way to the fixed points of the flow $\phi_{t}$ of the symplectic manifold $\left(\mathbb{R}^{2 n}, \omega_{0}\right)$ in time $t=1$ this thanks to the Hamiltonian differential equations via the generating functions.
\end{abstract}

Keywords: Symplectic Manifold, Generating functions, vector field.

Recibido: 18/10/2018. Aceptado: 18/12/2018. Publicado online: 31/12/2018.

${ }^{2}$ UNMSM, Facultad de Ciencias Matemáticas, e-mail: rgalvezp@unmsm.edu.pe 


\section{Introducción}

La Geometría y Topología Simpléctica tiene origen en la Física, precisamente en los espacios de fase y transformaciones canónicas de la Mecánica Clásica. Estudia propriedades de generalizaciones de esos espacios y transformaciones. El término simpléctico viene del griego symplektikos que significa "que entrelaza", "que une". Este nombre fue usado por primera vez en 1939 por H. Weyl. Con los trabajos de Lagrange sobre mecánica analítica en el siglo XIX se tiene inicios de la geometría simpléctica. De todas formas, durante el siglo XIX la naturaleza geométrica y cualitativa de la mecánica no estaba nada clara. Fue Poincaré, a principios del siglo XX, quien inicia la era cualitativa con el estudio de las órbitas en el problema de los n-cuerpos. Despues de Poincaré, las técnicas simplécticas empiezan a ser usadas y la geometría simpléctica va avanzando de manera que en los años 60 está consolidada como una rama de las matemáticas y el nexo de unión con la mecánica ha sido definitivamente establecido. Finalmente, en los últimos 30 años, la geometría simpléctica es una de las áreas de la matematica con mayor creciemiento en publicaciones e investigaciones.

\section{Preliminares}

Aqui presentaremos algunos conceptos previos sobre el origen de la Geometría Simpléctica y su origen relacionado con la Física. También presentaremos algunos resultados propios del análisis los cuales no demostraremos, el lector interesado puede encontrar la prueba en [3].

\subsection{Las ecuaciones de Hamilton via la ecuación de Newton}

La geometría simplectica se origino en el estudio de los sistemas hamiltonianos, que describen la evolución de sistemas mecanicos de naturaleza conservativa. Las ecuaciones que describen tales sistemas son llamadas ecuaciones de Hamilton y pueden ser derivadas directamente de la segunda Ley de Newton.

Vamos a considerar como ejemplo ilustrativo el movimiento de una particula de masa $m>0$ en $\mathbb{R}^{3}$ sometida a un campo de fuerzas conservativas $F$, dado en cada punto $q=\left(q_{1}, q_{2}, q_{3}\right) \in \mathbb{R}^{3}$ por

$$
F(q)=-\nabla V(q),
$$

donde $V: \mathbb{R}^{3} \longrightarrow \mathbb{R}$ es la energia potencial. Cada estado inicial, determinando por una posición y velocidad, determina completamente la trajectoria $q(t)$ de la particula atravez de la segunda Ley de Newton:

$$
m \ddot{q}(t)=-\nabla V(q(t)) .
$$

Sea $p=m \dot{q}$ el momento lineal de la particula. Podemos reescribir el sistema de 3 ecuaciones de segundo orden (1) como 6 ecuaciones de primer orden en las variables $q_{i}$ y $p_{i}$ :

$$
\dot{q}=\frac{p}{m}, \quad \dot{p}=-\nabla V .
$$

El espacio $\mathbb{R}^{3}=\left\{q=\left(q_{1}, q_{2}, q_{3}\right)\right\}$ de posibles posiciones de la particula es llamado el espacio de configuraciones, en cuanto el espacio $\mathbb{R}^{6}=\mathbb{R}^{3} \times \mathbb{R}^{3}=\{(q, p)\}$, consistiendo de posiciones y momentos es llamado espacio de face. Si denotamos la energia total de la particula por $H$,

$$
H(q, p)=\frac{\sum_{i=1}^{3} p_{i}^{2}}{2 m}+V(q),
$$

podemos escribir (1) en el espacio de face como

$$
\dot{q}_{i}=\frac{\partial H}{\partial p_{i}}, \quad \dot{p}_{i}=-\frac{\partial H}{\partial q_{i}} .
$$




\subsection{Abordaje Variacional}

Otra manera de obtener las ecuaciones de Hamilton es via las ecuaciones de Euler-Lagange, derivadas de principios variacionales. Un principio fundamental que rige la mecánica clásica es el principio de la acción mínima. Mas precisamente, considere un sistema cuyo espacio de configuraciones es $\mathbb{R}^{n}$, con coordenadas $q=\left(q_{1}, q_{2}, \ldots, q_{n}\right)$, de modo que el espacio de estados (i.e. posiciones y velocidades) sea $\mathbb{R}^{2 n}$, con coordenadas $(q, v)$.

Sea $L: \mathbb{R}^{2 n} \longrightarrow \mathbb{R}$ una función suave, llamada función lagrangiana. Dada una curva diferenciable $\gamma:[0, T] \longrightarrow \mathbb{R}^{n}$, definimos su acción por

$$
A_{L}(\gamma)=\int_{0}^{T} L(\gamma(t), \dot{\gamma}(t)) d t
$$

Fijando ahora dos puntos $q_{0}$ y $q_{1}$ en $\mathbb{R}^{n}$ y denotando por $\mathcal{C}\left([0, T], q_{0}, q_{1}\right)$ el conjunto de curvas suaves $\gamma:[0, T] \longrightarrow \mathbb{R}^{n}$ tales que $\gamma(0)=q_{0}$ y $\gamma(T)=q_{1}$. Buscamos en este conjunto puntos críticos para el funcional de acción $A_{L}$, o sea, curvas $\gamma$ para las cuales

$$
\frac{d}{d s} A_{L}\left(\Gamma_{s}\right)_{\left.\right|_{s=0}}=0
$$

donde $\Gamma_{s} \in \mathcal{C}\left([0, T], q_{0}, q_{1}\right), s \in(-\epsilon, \epsilon)$, es una variación diferenciable de curvas tal que $\Gamma_{0}=\gamma$.

Proposición 2.1 Una curva $\gamma$ es un punto crítico de $A_{L}$ si y solamente sí satisface la ecuación de Euler-Lagrange

$$
\frac{d}{d t} \frac{\partial L}{\partial v}(\gamma(t), \dot{\gamma}(t))=\frac{\partial L}{\partial q}(\gamma(t), \dot{\gamma}(t))
$$

Diferentes funciones lagrangianas corresponden a diferentes sistemas físicos, y la evolución de cada sistema es descrita por las soluciones de las ecuaciones de Euler-Lagrange asociadas.

Veremos ahora como un cambio de variables pueden transformar las ecuaciones de EulerLagrange en las ecuaciones de Hamilton. Considere la aplicación $\mathbb{F L}: \mathbb{R}^{2 n} \longrightarrow \mathbb{R}^{2 n}$, dada por

$$
\mathbb{F} \mathbb{L}\left(q_{1}, q_{2}, \ldots, q_{n}, v_{1}, \ldots, v_{n}\right)=\left(q_{1}, \ldots, q_{n}, \frac{\partial L}{\partial v_{1}}(q, v), \ldots ., \frac{\partial L}{\partial v_{n}}(q, v)\right)
$$

llamada transformada de Legendre asociada a $L$. suponiendo que $\mathbb{F} \mathbb{L}$ sea un difeomorfismo, obtenemos nuevas coordenadas $(q, p) \in \mathbb{R}^{2 n}$ donde $p=\frac{\partial L}{\partial v}$ es llamado momento generalizado. Definimos la hamiltomiana $H$ asociada a $L$ como

$$
H(q, p)=\sum_{i=1}^{n} p_{i} v_{i}-L(q, v)
$$

tenemos entonces que

$$
\begin{aligned}
& \frac{\partial H}{\partial q_{i}}=\sum_{j=1}^{n} p_{j} \frac{\partial v_{j}}{\partial q_{i}}-\frac{\partial L}{\partial q_{i}}-\sum_{j=1}^{n} \frac{\partial L}{\partial v_{j}} \frac{\partial v_{j}}{\partial q_{i}}=-\frac{\partial L}{\partial q_{i}} \\
& \frac{\partial H}{\partial p_{i}}=v_{i}+\sum_{j=1}^{n} p_{j} \frac{\partial v_{j}}{\partial p_{i}}-\frac{\partial L}{\partial v_{j}} \frac{\partial v_{j}}{\partial p_{i}}=v_{i} .
\end{aligned}
$$

consecuentemente, las ecuaciones de Euler-Lagrange son equivalentes a las ecuaciones de Hamilton

$$
\frac{d q_{i}}{d t}=\frac{\partial H}{\partial p_{i}}, \quad \frac{d p_{i}}{d t}=-\frac{\partial H}{\partial q_{i}} .
$$




\subsection{Geometrización de las ecuaciones de Hamilton}

como vimos antes las ecuaciones de Hamilton pueden ser derivadas de las ecuaciones de Newton e de Euler-Lagrange. En esta sección colocaremos las ecuaciones de Hamilton en un contexto geométrico atravez de una formulación intrinseca.

Considere el espacio de face $\mathbb{R}^{2 n}$, con coordenadas $\left(q_{1}, \ldots, q_{n}, p_{1}, \ldots, p_{n}\right)$. La elección de cualquier función $H \in \mathcal{C}^{\infty}\left(\mathbb{R}^{2 n}\right)$ determina un campo hamiltoniano

$$
X_{H}:=-J_{0} \nabla H=\sum_{i=1}^{3} \frac{\partial H}{\partial p_{i}} \frac{\partial}{\partial q_{i}}-\frac{\partial H}{\partial q_{i}} \frac{\partial}{\partial p_{i}}
$$

donde $J_{0}$ es una matriz $2 n \times 2 n$ dada por

$$
J_{0}=\left(\begin{array}{cc}
0 & -I \\
I & 0
\end{array}\right)
$$

La función $H$ es llamada hamiltoniana, y las ecuaciones de Hamilton (2) asumen la forma

$$
\dot{c}(t)=X_{H}(c(t))
$$

donde $c(t)=\left(q_{1}(t), \ldots, q_{n}(t), p_{1}(t), \ldots, p_{n}(t)\right)$. Note que $H$ es siempre preservado a lo largo de las soluciones de $(5)$ :

$$
\frac{d}{d t} H(c(t))=\nabla H(c(t)) \cdot \dot{c}(t)=-\nabla H(c(t)) . J_{0} \nabla H(c(t))=0 .
$$

Esa propiedad y algunas mas dan al formalismo hamiltoniano su caracter conservativo.

\subsection{Variedades Simplécticas}

Definición 2.2 Una variedad simpléctica es un par $(M, \omega)$ donde $M$ es una variedad diferenciable y $\omega$ es una 2-forma definida en $M$ tal que es cerrada y no degenerada. Es decir:

- $d \omega=0$, donde d es la derivada exterior

- Si $\omega_{p}(u, v)=0 \forall \in v \in T_{p} M$ con $p \in M \Rightarrow u=0$.

Una 2-forma satisfaciendo estas dos condiciones es llamada forma simpléctica. La 2-forma $\omega$ asi definida hace que la variedad sea siempre de dimensión par.

Ejemplo 2.3 Sea $M=\mathbb{R}^{2 n}$ con coordenadas lineales $x_{1}, x_{2}, \ldots, x_{n}, y_{1}, \ldots, y_{n}$. La 2-forma

$$
\omega_{0}=\sum_{i=1}^{n} d x_{i} \wedge d y_{i}
$$

es simpléctica y el conjunto

$$
\left\{\left(\frac{\partial}{\partial x_{1}}\right)_{p}, \ldots \ldots,\left(\frac{\partial}{\partial x_{n}}\right)_{p},\left(\frac{\partial}{\partial y_{1}}\right)_{p}, \ldots \ldots,\left(\frac{\partial}{\partial y_{n}}\right)_{p}\right\}
$$

es una base simpléctica de $T_{p} M$. 


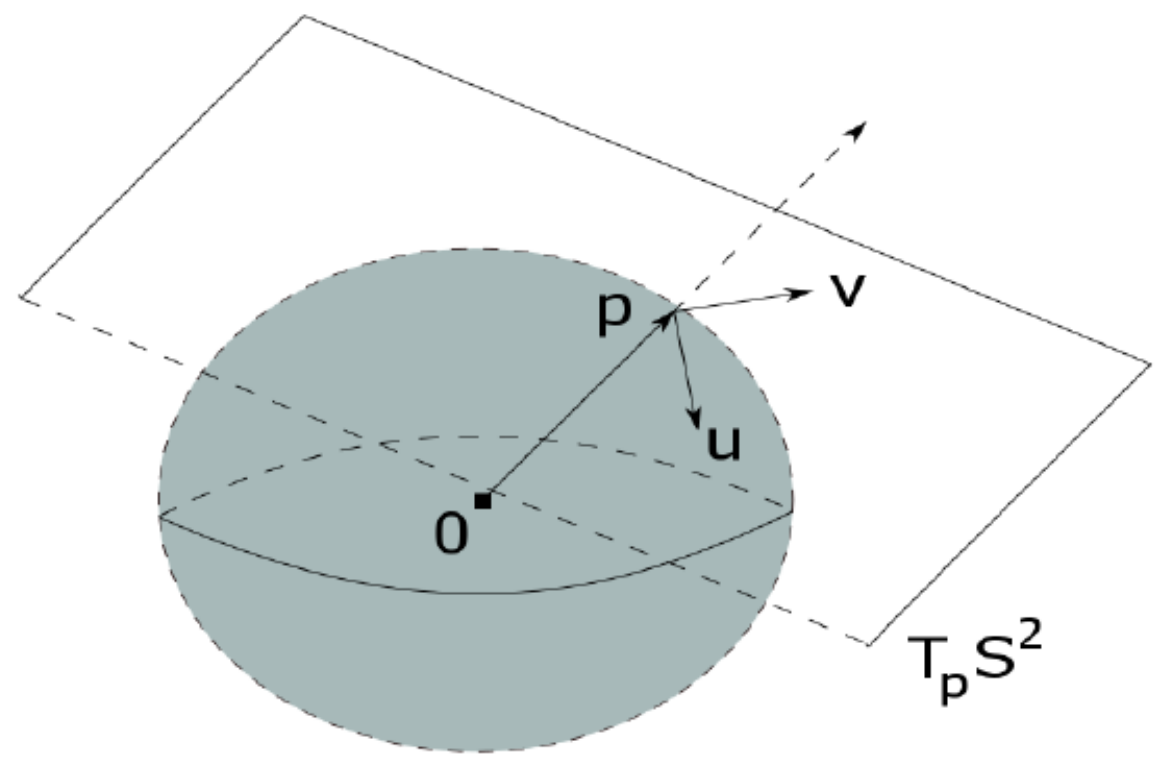

Figura 1: Esfera $S^{2} \subset \mathbb{R}^{\nVdash}$

Ejemplo 2.4 Sea $S^{2}=\left\{(x, y, z) \in \mathbb{R}^{3} \mid x^{2}+y^{2}+z^{2}=1\right\}$ la esfera unitaria en $\mathbb{R}^{3}$. Los vectores tangentes a $S^{2}$ en un punto $p \in S^{2}$ pueden ser identificados con los vectores ortogonales a $p$. Es decir

$$
T_{p} S^{2}=\left\{u \in \mathbb{R}^{3} \mid\langle u, p\rangle=0\right\} .
$$

La forma simpléctica estandar en $S^{2}$ es inducida por el producto interno y el producto exterior en $\mathbb{R}^{3}$

$$
\omega_{p}(u, v):=\langle p, u \times v\rangle, \quad \forall u, v \in T_{p} S^{2} .
$$

Esta forma es cerrada por que es de grado máximo en $S^{2}$ y es no degenerada pues $\langle p, u \times v\rangle \neq 0$ cuando $u \neq 0$.

Definición 2.5 Sean $\left(M_{1}, \omega_{1}\right)$ y $\left(M_{2}, \omega_{2}\right)$ dos variedades simplécticas de la misma dimensión y sea $\varphi: M_{1} \rightarrow M_{2}$ un difeomorfismo. Entonces $\varphi$ es un simplectomorfismo si $\varphi^{*} \omega_{2}=\omega_{1}$.

Donde $\varphi^{*}$ es el pull-back de $\varphi$, es $\operatorname{decir} \varphi^{*}: \Lambda^{2}\left(M_{2}\right) \rightarrow \Lambda^{2}\left(M_{1}\right)$ definido como

$$
\varphi^{*} \omega_{2}(p):=\omega_{2}(p)\left(d \varphi_{p}(u), d \varphi_{p}(v)\right) \quad \forall p \in M_{1}, \quad \forall u, v \in T_{p} M_{1} .
$$

Problema: ¿Como distinguir dos variedades simplécticas? Intentar encontrar invariantes de la forma simpléctica. El siguiente teorema nos dice que no existen invariantes locales.

Proposición 2.6 (Teorema de Darboux) Si $(M, \omega)$ es una variedad simpléctica entonces para todo $x \in M$ existe una vecindad $U_{x}$ simplectomorfa a un abierto de $\left(\mathbb{R}^{2 n}, \omega_{0}\right)$, donde $\omega_{0}=$ $d x_{i} \wedge d y_{i}$

Demostración. Considerando una carta local de $M$ en torno de $p$, podemos asumir que $M=$ $\mathbb{R}^{2 n}$ y $p=0 \in \mathbb{R}^{2 n}$, sin perdida de generalidad. Ademas por una transformación lineal $L \in \mathbb{R}^{2 n}$ podemos también asumir que $\omega_{0}=\sum_{i=1}^{n} d q_{i} \wedge d p_{i}$ donde $q_{i}, p_{i}$ son las coordenadas en $\mathbb{R}^{2 n}$.

De esta forma tenemos dos formas simplécticas $\omega_{0}$ y $\omega$ definidas en una vecindad $\mathcal{U}$ de $0 \in \mathbb{R}^{2 n}$ tales que $\left(\omega-\left.\omega_{0}\right|_{0}=0\right.$. Usando 
Lema 2.7 (Lema de Poincaré) Sea $U \subset M$ abierto y contractible. Entonces toda forma diferenciable cerrada definida en $U$ es exacta.

se tiene que existen $\mathcal{U}_{0} \subset \mathcal{U}$ vecindad de 0 y 1 -forma $\mu$ definida em $\mathcal{U}_{0}$, tales que $\left.\left(\omega_{0}-\omega\right)\right|_{\mathcal{U}_{0}}=d \mu$ y $\left.\mu\right|_{0}=0$. Sea

$$
\Omega_{t}=(1-t) \omega+t \omega_{0}=\omega+t d \mu, \quad t \in[0,1] .
$$

Como $\left.d \mu\right|_{0}=0$, podemos disminuir $\mathcal{U}_{0}$, si es necesario, de modo que $\Omega_{t}$ sea simplectica em $\mathcal{U}_{0}$ para todo $t \in[0,1]$. Considere el único campo de vectores tiempo-dependientes $X_{t}, t \in[0,1]$ en $\mathcal{U}_{0}$, satisfaciendo

$$
i_{X_{t}} \Omega_{t}=-\mu
$$

para todo $t \in[0,1]$. Como $\Omega_{t}$ es simpléctica, $X_{t}$ esta bien definida y depende suavemente de $t$. Ahora observe que $\left.X_{t}\right|_{0}=0$, para todo $t \in[0,1]$. Por lo tanto, para $\mathcal{U}_{1} \subset \mathcal{U}_{0}$ suficientemente pequeña, podemos integrar $X_{t}$ hasta $t=1$ y obtener una isotopia $\rho_{t}: \mathcal{U}_{1} \rightarrow \mathcal{U}_{0}, t \in[0,1]$ esto es $\rho_{0}=I d$ y $\frac{d \rho_{t}}{d t}=X_{t} \circ \rho_{t}$. Tenemos entonces

$$
\frac{d}{d t} \rho_{t}^{*} \Omega_{t}=\rho_{t}^{*}\left(\frac{d \Omega_{t}}{d t}+\mathcal{L}_{X_{t}} \Omega_{t}\right)=\rho_{t}^{*}\left(d \mu+d i_{X_{t}} \Omega_{t}\right)=0 .
$$

Se sigue que $\rho_{1}: \mathcal{U}_{1} \rightarrow \rho_{1}\left(\mathcal{U}_{1}\right) \subset \mathcal{U}_{0}$ tal que $\rho_{1}^{*} \omega_{0}=\rho_{0}^{*} \omega=\omega$ y $\rho_{1}(0)=0$. Tomando $U:=\mathcal{U}_{1}$ y $V:=\rho_{1}\left(\mathcal{U}_{1}\right)$ se concluye la demostración.

Corolario 2.8 Sean $\left(M_{1}, \omega_{1}\right)$ y $\left(M_{2}, \omega_{2}\right)$ dos variedades Simplécticas de dimensión 2n. Dados $p_{1} \in M_{1}$ y $p_{2} \in M_{2}$, existen vecindades $V_{1} \subset M_{1}$ de $p_{1}, V_{2} \subset M_{2}$ de $p_{2}$ y un simpléctomorfismo $\varphi: V_{1} \rightarrow V_{2}$ satisfaciendo $\varphi\left(p_{1}\right)=p_{2}$ y $\varphi^{*} \omega_{2}=\omega_{1}$.

\subsection{Funciones Generadoras}

Considere la variedade simpléctica $\left(\mathbb{R}^{2 n}, \omega_{0}\right)$ com 2 -forma estandar $\omega_{0}=\Sigma_{i=1}^{n} d x^{i} \wedge d y^{i}$ y sea la Hamiltoniana tiempo-dependiente $H_{t}: \mathbb{R}^{2 n} \longrightarrow \mathbb{R}$ con período 1 , es decir $H_{t+1}=H_{t}$ la cual genera el campo tiempo-dependiente $X_{H_{t}}$, por la ecuación

$$
\omega_{0}\left(X_{H_{t}}, *\right)=d H_{t}(*) .
$$

El campo $X_{H_{t}}$ genera la isotopia $\varphi_{H}^{t}$ por la ecuación

$$
\frac{d}{d t} \varphi_{H}^{t}=X_{H_{t}} \circ \varphi_{H}^{t}
$$

Nosotros usamos $(x, y)$ para indicar el splitting $\mathbb{R}^{2 n}=\left(\mathbb{R}^{n} \times 0\right) \oplus\left(0 \times \mathbb{R}^{n}\right) \simeq \mathbb{R}^{n} \times \mathbb{R}^{n} \mathrm{e}$ identificamos $\mathbb{R}^{2 n} \simeq \mathbb{C}^{n}$ por el isomorfismo $(x, y) \rightarrow x+i y$. Se quiere estudiar la dinamica del $\operatorname{mapa} \varphi_{H}^{1}=\phi$.

Sea $\psi: \mathbb{R}^{2 n} \longrightarrow \mathbb{R}^{2 n}$ un simplectomorfismo global tal que $\psi(x, y)=(X, Y)$ donde

$$
X=u(x, y), \quad Y=v(x, y) .
$$

Se dice que $\psi$ es $\mathcal{C}^{1}$-próximo de la identidade, si la primera derivada de $\psi$ satisface

$$
\|d \psi(z)-\mathbb{I}\| \leq \frac{1}{2}
$$

para todo $z \in \mathbb{R}^{2 n}$. 


\section{Proposición 2.9 [Teorema de la desigualdad del valor medio]}

Sea $U \subset \mathbb{R}^{m}$ abierto y conexo. Si $f: U \subset \mathbb{R}^{m} \longrightarrow \mathbb{R}^{n}$ es $\mathcal{C}^{\infty}$ con $\left\|f^{\prime}(x)\right\| \leq M$ para todo $x \in U$, entonces $f$ es Lipchitziana con constante $M$

$$
\|f(y)-f(x)\| \leq M|y-x|
$$

para todo $x, y \in U$.

\section{Proposición 2.10 [Teorema de Perturbación de la identidad]}

Sea $\varphi: U \subset \mathbb{R}^{m} \longrightarrow \mathbb{R}^{m}$ una contracción definida en $U$ abierto. La aplicación

$f: U \subset \mathbb{R}^{m} \longrightarrow \mathbb{R}^{m}$ dada por $f(x)=x+\varphi(x)$ es un homeomorfismo de $U$ sobre $f(U)$.

Además si $U=\mathbb{R}^{m}$ se tiene $f(U)=\mathbb{R}^{m}$.

Lema 2.11 Si $\psi$ es $\mathcal{C}^{1}$-próxima de la identidade, entonces existe una función $\mathcal{C}^{\infty}, S=S(x, y)$ en $\mathbb{R}^{2 n}$ tal que $\psi(x, y)=(X, Y)$ si y solamente si

$$
\begin{aligned}
& X-x=\frac{\partial}{\partial Y} S(x, Y) \\
& y-Y=\frac{\partial}{\partial x} S(x, Y) .
\end{aligned}
$$

\section{Demostración.}

Consideremos $\psi(x, y)=(X, Y)$ asi como antes y sea

$$
\begin{aligned}
\varpi: \mathbb{R}^{2 n} \longrightarrow \mathbb{R}^{2 n} \\
\quad(x, y) \longrightarrow(x, v(x, y))
\end{aligned}
$$

Afirmación: $\varpi$ tiene una inversa global.

En efecto

$$
\varpi(x, y)=\varphi(x, y)+I d(x, y)
$$

donde $\varphi(x, y)=(0, v(x, y)-y)$

Primero: $\varpi$ es $\mathcal{C}^{1}$-próximo de la identidade.

Por la hipotesis, se tiene $\|d \psi(z)-\mathbb{I}\| \leq \frac{1}{2}$ para todo $z \in \mathbb{R}^{2 n}$, es decir la matriz

$$
d \psi(z)-\mathbb{I}=\left(\begin{array}{cc}
\frac{\partial}{\partial x} u(z)-\mathbb{I} & \frac{\partial}{\partial y} u(z) \\
\frac{\partial}{\partial x} v(z) & \frac{\partial}{\partial y} v(z)-\mathbb{I}
\end{array}\right)
$$

tiene norma menor que $\frac{1}{2}$. Por otro lado

$$
d \varpi(z)-\mathbb{I}=\left(\begin{array}{cc}
0 & 0 \\
\frac{\partial}{\partial x} v(z) & \frac{\partial}{\partial y} v(z)-\mathbb{I}
\end{array}\right)
$$

es claro que $\|d \varpi(z)-\mathbb{I}\| \leq\|d \psi(z)-\mathbb{I}\|$, luego

$$
\|d \varpi(z)-\mathbb{I}\| \leq \frac{1}{2}
$$

para todo $z \in \mathbb{R}^{2 n}$. Por tanto $\varpi$ es $\mathcal{C}^{1}$ próximo de la identidad.

Segundo: $\varphi$ es Lipchitziana (contracción) 
En efecto, derivando $\varphi(x, y)=(0, v(x, y)-y)$ se tiene

$$
d \varphi(x, y)=\left(\begin{array}{cc}
0 & 0 \\
\frac{\partial}{\partial x} v(x, y) & \frac{\partial}{\partial y} v(x, y)-\mathbb{I}
\end{array}\right)
$$

luego $\|d \varphi(x, y)\| \leq\|d \psi(x, y)-\mathbb{I}\| \leq \frac{1}{2}$, por el Teorema de la desigualdad del valor medio, tenemos

$$
\left\|\varphi(z)-\varphi\left(z_{0}\right)\right\| \leq \frac{1}{2}\left|z-z_{0}\right|
$$

para todo $z, z_{0} \in \mathbb{R}^{2 n}$.

Por el Teorema de perturbación de la identidad, se tiene

$$
\varpi(x, y)=\varphi(x, y)+I d(x, y)
$$

para todo $(x, y) \in \mathbb{R}^{2 n}$ com $\varphi$ una $\frac{1}{2}$-contracción en $\mathbb{R}^{2 n}$, entonces $\varpi$ es un homeomorfismo, mas como $\varpi(x, y)=(x, v(x, y))$ es de clase $\mathcal{C}^{\infty}$, se tiene que $\varpi$ es um difeomorfismo en todo $\mathbb{R}^{2 n}$.

Por otro lado como $\varpi$ tiene inversa global, existen aplicaciones $\mathcal{C}^{\infty}$

$$
\begin{gathered}
\mathbb{R}^{2 n} \longrightarrow \mathbb{R}^{2 n} \\
(x, Y) \longrightarrow g(x, Y)
\end{gathered}
$$

tal que $y=g(x, Y)=g(x, v(x, y))$ y también

$$
\begin{gathered}
\mathbb{R}^{2 n} \longrightarrow \mathbb{R}^{2 n} \\
(x, Y) \longrightarrow f(x, Y)
\end{gathered}
$$

tal que $X=f(x, Y)=u(x, g(x, Y))$.

Pues la inversa de $\varpi$ es definida como

$$
\begin{aligned}
\varpi^{-1}: \mathbb{R}^{2 n} & \longrightarrow \mathbb{R}^{2 n} \\
(x, Y) & \longrightarrow(x, g(x, Y))
\end{aligned}
$$

En efecto $\varpi \circ \varpi^{-1}(x, Y)=\varpi(x, g(x, Y))=(x, v(x, g(x, Y)))=(x, v(x, y))=(x, Y)$ y también $\varpi^{-1} \circ \varpi(x, y)=\varpi^{-1}(x, v(x, y))=(x, g(x, v(x, y)))=(x, y)$.

Desde que $\psi$ es un simplectomorfismo la 1 -forma

$$
y d x+X d Y=g d x+f d Y
$$

es cerrada, pues tomando la derivada exterior a esta 1-forma tenemos $d y \wedge d x+d X \wedge d Y=0$ desde que $\psi^{*}(d X \wedge d Y)=d X \wedge d Y=d x \wedge d y$.

Por tanto existe una función, (por el hecho que $H_{d R}^{1}\left(\mathbb{R}^{2 n}\right)=0$ ) $W: \mathbb{R}^{2 n} \longrightarrow \mathbb{R}$ tal que

$$
\begin{aligned}
X & =f(x, Y)=\frac{\partial}{\partial Y} W(x, Y) \\
y & =g(x, Y)=\frac{\partial}{\partial x} W(x, Y)
\end{aligned}
$$

Luego la función

$$
S(x, Y)=W(x, Y)-\langle x, Y\rangle
$$

satisface

$\mathrm{y}$

$$
\frac{\partial}{\partial Y} S(x, Y)=\frac{\partial}{\partial Y} W(x, Y)-x=X-x
$$

$$
\frac{\partial}{\partial x} S(x, Y)=\frac{\partial}{\partial x} W(x, Y)-Y=y-Y .
$$




\subsection{Acción Simpléctica Discreta}

Consideremos ahora $N \gg 1$ (suficientemente grande), tal que

$$
\phi=\varphi_{H}^{1}=\left(\varphi_{H}^{1} \circ\left(\varphi_{H}^{\frac{N-1}{N}}\right)^{-1}\right) \circ\left(\varphi_{H}^{\frac{N-1}{N}} \circ\left(\varphi_{H}^{\frac{N-2}{N}}\right)^{-1}\right) \circ \ldots \ldots \ldots \circ\left(\varphi_{H}^{\frac{1}{N}} \circ\left(\varphi_{H}^{0}\right)^{-1}\right)
$$

denotemos

$$
\psi_{j}=\varphi_{H}^{\frac{j}{N}} \circ\left(\varphi_{H}^{\frac{j-1}{N}}\right)^{-1}
$$

para todo $j \in\{1,2, \ldots, N\}$.

Luego

$$
\phi=\psi_{N} \circ \psi_{N-1} \circ \ldots \ldots \ldots \ldots . . . . \psi_{1}
$$

como $N$ es suficientemente grande se tiene que $\psi_{j}$ es $\mathcal{C}^{1}$-próximo de la identidad. Por tanto para cada $j$ existe uma función $\mathcal{C}^{\infty}, S_{j}: \mathbb{R}^{2 n} \longrightarrow \mathbb{R}$ la cual genera los simplectomorfismos $\psi_{j}\left(x_{j}, y_{j}\right)=\left(x_{j+1}, y_{j+1}\right)$ via las ecuaciones diferenciales Hamiltonianas

$$
x_{i}-x_{i-1}=\frac{\partial}{\partial y_{i}} S_{i-1}\left(x_{i-1}, y_{i}\right) \quad y_{i}-y_{i+1}=\frac{\partial}{\partial x_{i}} S_{i}\left(x_{i}, y_{i+1}\right) .
$$

Sabemos que las soluciones de (6) pueden ser descritas como um problema variacional. Denotemos por $\mathcal{P}=\left(\mathbb{R}^{2 n}\right)^{N}$ el conjunto de caminos discretos $\xi=\left(z_{1}, z_{2}, \ldots \ldots, z_{N}\right)$ donde $z_{j}=\left(x_{j}, y_{j}\right) \in \mathbb{R}^{2 n}$. Definamos, Acción Simpléctica Discreta

$$
\mathbb{A}_{H, N}: \mathcal{P} \longrightarrow \mathbb{R}
$$

por la formula

$$
\mathbb{A}_{H, N}\left(z_{1}, \ldots, z_{N}\right)=\sum_{i=1}^{N}\left[\left\langle x_{i}, y_{i+1}-y_{i}\right\rangle+S_{i}\left(x_{i}, y_{i+1}\right)\right]
$$

para $\left(z_{1}, \ldots, z_{N}\right) \in \mathbb{R}^{2 n N}$ donde estamos considerando $(i$ mód $N)$.

Lema 2.12 Un punto $\xi \in \mathcal{P}$ es crítico para $\mathbb{A}_{H, N}$ si y solamente si $\xi$ satisface las ecuaciones diferenciales Hamiltonianas

$$
\begin{aligned}
y_{i}-y_{i+1} & =\frac{\partial}{\partial x_{i}} S_{i}\left(x_{i}, y_{i+1}\right) \\
x_{i}-x_{i-1} & =\frac{\partial}{\partial y_{i}} S_{i-1}\left(x_{i-1}, y_{i}\right) .
\end{aligned}
$$

para todo $i=\{1,2, \ldots \ldots, N\} \operatorname{com}(i \bmod N)$.

\section{Demostración.}

$$
\begin{aligned}
\mathbb{A}_{H, N}(\xi) & =\left\langle x_{1}, y_{2}-y_{1}\right\rangle+S_{1}\left(x_{1}, y_{2}\right)+\left\langle x_{2}, y_{3}-y_{2}\right\rangle+S_{2}\left(x_{2}, y_{3}\right) \\
\cdot & \cdot \\
& +\left\langle x_{i-1}, y_{i}-y_{i-1}\right\rangle+S_{i-1}\left(x_{i-1}, y_{i}\right)+\left\langle x_{i}, y_{i+1}-y_{i}\right\rangle+S_{i}\left(x_{i}, y_{i+1}\right)
\end{aligned}
$$




$$
+\left\langle x_{N-1}, y_{N}-y_{N-1}\right\rangle+S_{N-1}\left(x_{N-1}, y_{N}\right)+\left\langle x_{N}, y_{1}-y_{N}\right\rangle+S_{N}\left(x_{N}, y_{1}\right) .
$$

Derivando con respecto de las variables $x_{i}$ e $y_{i}$ se tiene

$$
\begin{gathered}
\frac{\partial}{\partial x_{1}} \mathbb{A}_{H, N}(\xi)=y_{2}-y_{1}+\frac{\partial}{\partial x_{1}} S_{1}\left(x_{1}, y_{2}\right) \\
\frac{\partial}{\partial y_{1}} \mathbb{A}_{H, N}(\xi)=x_{N}-x_{1}+\frac{\partial}{\partial y_{1}} S_{N}\left(x_{N}, y_{1}\right) \\
\cdot \\
\cdot \\
\frac{\partial}{\partial x_{i}} \mathbb{A}_{H, N}(\xi)=y_{i+1}-y_{i}+\frac{\partial}{\partial x_{i}} S_{i}\left(x_{i}, y_{i+1}\right) \\
\frac{\partial}{\partial y_{i}} \mathbb{A}_{H, N}(\xi)=x_{i-1}-x_{i}+\frac{\partial}{\partial y_{i}} S_{i-1}\left(x_{i-1}, y_{i}\right) \\
\cdot \cdot \\
\frac{\partial}{\partial x_{N}} \mathbb{A}_{H, N}(\xi)=y_{1}-y_{N}+\frac{\partial}{\partial x_{N}} S_{N}\left(x_{N}, y_{1}\right) \\
\frac{\partial}{\partial y_{N}} \mathbb{A}_{H, N}(\xi)=x_{N-1}-x_{N}+\frac{\partial}{\partial y_{N}} S_{N-1}\left(x_{N-1}, y_{N}\right) .
\end{gathered}
$$

Luego si $\xi$ es un punto crítico de $\mathbb{A}_{H, N}$ se tiene que todas las ecuaciones anteriores son nulas,

$$
\frac{\partial}{\partial x_{i}} \mathbb{A}_{H, N}(\xi)=y_{i+1}-y_{i}+\frac{\partial}{\partial x_{i}} S_{i}\left(x_{i}, y_{i+1}\right)=0
$$

$\mathrm{e}$

$$
\frac{\partial}{\partial y_{i+1}} \mathbb{A}_{H, N}(\xi)=x_{i}-x_{i+1}+\frac{\partial}{\partial y_{i+1}} S_{i}\left(x_{i}, y_{i+1}\right)=0
$$

Lema 2.13 Una sucesión periódica $\xi$ es un punto crítico de $\mathbb{A}_{H, N}: \mathcal{P}_{\text {per }} \longrightarrow \mathbb{R}$ si y solamente si $z_{1}=\left(x_{1}, y_{1}\right)$ es un punto fijo de $\phi=\varphi_{H}^{1}$ y $\psi_{j}\left(z_{j}\right)=z_{j+1}=\varphi_{H}^{\frac{j}{N}}\left(z_{1}\right)$ para todo $j=\{1,2,3, \ldots ., N\}$.

Demostración. Como $\xi$ es un punto crítico de $\mathbb{A}_{H, N}$ por el Lema 2.1 satisfacen las siguientes ecuaciones

$$
\begin{aligned}
y_{i}-y_{i+1} & =\frac{\partial}{\partial x_{i}} S_{i}\left(x_{i}, y_{i+1}\right) \\
x_{i+1}-x_{i} & =\frac{\partial}{\partial y_{i+1}} S_{i}\left(x_{i}, y_{i+1}\right)
\end{aligned}
$$

para todo $i \in\{1,2, \ldots \ldots, N\}$ con $(i \bmod N)$. Luego por el Lema 1.3, si y solamente si $\psi_{j}$ é $\mathcal{C}^{1}$-próximo de la identidad, entonces existe una funcion $S_{j}: \mathbb{R}^{2 n} \longrightarrow \mathbb{R}$ tal que

$$
\psi_{j}\left(x_{j}, y_{j}\right)=\left(x_{j+1}, y_{j+1}\right) .
$$

Luego como $\psi_{j}=\varphi_{H}^{\frac{j}{N}} \circ\left(\varphi_{H}^{\frac{j-1}{N}}\right)^{-1}$ para todo $j=\{1,2, \ldots \ldots, N\}$.

Para $j=1$ se tiene

$$
\psi_{1}\left(z_{1}\right)=\varphi_{H}^{\frac{1}{N}} \circ\left(\varphi_{H}^{0}\right)^{-1}=\varphi_{H}^{\frac{1}{N}}\left(z_{1}\right)
$$


de donde obtenemos

$$
\varphi_{H}^{\frac{1}{N}}\left(z_{1}\right)=z_{2}
$$

Para $j=2$ se tiene

$$
\psi_{2}\left(z_{2}\right)=\varphi_{H}^{\frac{2}{N}} \circ\left(\varphi_{H}^{\frac{1}{N}}\right)^{-1}\left(z_{2}\right)=\varphi_{H}^{\frac{2}{N}}\left(z_{1}\right)
$$

de donde obtenemos

$$
\varphi_{H}^{\frac{2}{N}}\left(z_{1}\right)=z_{3}
$$

siguiendo de la misma manera se tiene

$$
\varphi_{H}^{\frac{j}{N}}\left(z_{1}\right)=z_{j+1}
$$

para todo $j \in\{1,2, \ldots . ., N\}$.

Finalmente $z_{1}$ es punto fijo de $\phi$, en efecto

$$
\phi\left(z_{1}\right)=\psi_{N} \circ \psi_{N-1} \circ \ldots \ldots \circ \psi_{1}\left(z_{1}\right)
$$

como $\psi\left(z_{j}\right)=z_{j+1}$ se tiene

$$
\phi\left(z_{1}\right)=\psi_{N} \circ \psi_{N-1}\left(z_{N-1}\right)=\psi_{N}\left(z_{N}\right)=z_{N+1}=z_{1}
$$

lo cual muestra el lema.

\section{Resultados}

Los resultados presentados aqui nos muestran la relación biunívoca entre los puntos críticos de un funcional y los puntos fijos de un flujo asociado a este funcional, eso es bastante importante porque nos permite resolver problemas desde el punto de vista de puntos fijos de funciones diferenciables y llevarlos via esta relación al espacio de Hilber $\mathcal{P}=\left(\mathbb{R}^{2 n}\right)^{N}$ con $N>>1$ bastante grande lo cual nos permite caracterizar los puntos críticos de $\mathbb{A}_{H, N}$ con alguna propiedad adicional.

\section{Discusión}

Lo presentado hasta aqui nos brinda resultados que relacionan puntos críticos de funcionales y puntos fijos de flujos diferenciales, como todo esto es cierto siempre que se considere $N$ suficientemente grande pues en ese caso se tiene que los $\psi_{j}$ son $\mathcal{C}^{1}$-próximos de la identidad y a partir de ello se obtiene dicho resultado, la pregunta sería ¿que propiedad adicional se obtiene en el caso en $N \rightarrow \infty$ ? ¿Seguiran siendo valido estos Lemas? o ¿se obtendra mejores resultados?

\section{Conclusión}

En este trabajo presentamos algunas de las principales herramientas de la geometría simpléctica y las relacionamos con el análisis y la dinámica de los flujos generados por un campo vectorial, con esto queremos invitar al lector a que es posible relacionar diversas áreas de las matemáticas e interactuar unas con otras para conseguir resultados bastantes provechosos en la investigación. 


\section{Referencias bibliográficas}

[1] Bursztyn, H. y Macarini, L.(2006). Introdução a Geometria Simplética. Recuperado de http://w3.impa.br/ henrique/papers/EGD2806.pdf

[2] Hofer, H. and Zehnder, E.(2010). Symplectic Invariants and Hamiltonian Dynamics. Berlin, Alemania: Birkhäuser .

[3] Elon, L.(2007) Curso de análise Vol. 2. Rio de Janeiro. Brasil: Projeto Euclides IMPA, .

[4] John, M. Lee. (1997). Riemannian Manifolds An Introduction to Curvature. New York, United State of America: Springer-Verlang.

[5] McDuff, D. and Salamon, D.(1998) Introduction to Symplectic Topology. New York, United State of America: Oxford University Press Math.

[6] McDuff D. and Salamon D.(2004). J-holomorphic curves and symplectic topology. Providence, Rhode Island: American Mathematical Society. 